\section{BRAZIULIAN JOURNAL}

OF MEDICAL AND BIOLOGICAL RESHARCH

www.bjournal.com.br
ISSN 0100-879X

Volume 43 (03) 226-324 March 2010

BIOMEDICAL SCIENCES

AND

CLINICAL INVESTIGATION

Braz J Med Biol Res, March 2010, Volume 43(3) 285-289

Effect of escitalopram on the processing of emotional faces

W.C. Alves-Neto, V.G. Guapo, F.G. Graeff, J.F.W. Deakin and C.M. Del-Ben

The Brazilian Journal of Medical and Biological Research is partially financed by
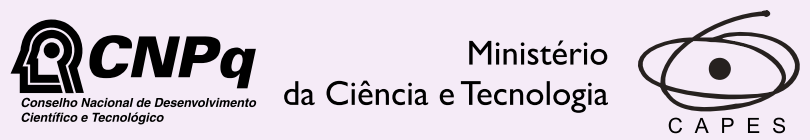

Ministério da Educação
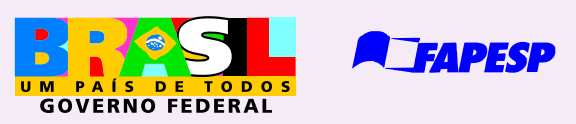

Institutional Sponsors 


\title{
Effect of escitalopram on the processing of emotional faces
}

\author{
W.C. Alves-Neto ${ }^{1}$, V.G. Guapo ${ }^{1}$, F.G. Graeff ${ }^{1}$, J.F.W. Deakin² and C.M. Del-Ben ${ }^{1}$ \\ ${ }^{1}$ Divisão de Psiquiatria, Departamento de Neurociências e Ciências do Comportamento, \\ Faculdade de Medicina de Ribeirão Preto, Universidade de São Paulo, Ribeirão Preto, SP, Brasil \\ ${ }^{2}$ Neuroscience and Psychiatry Unit, School of Medicine, The University of Manchester, Manchester, UK
}

\begin{abstract}
Serotonin has been implicated in the neurobiology of depressive and anxiety disorders, but little is known about its role in the modulation of basic emotional processing. The aim of this study was to determine the effect of the selective serotonin reuptake inhibitor, escitalopram, on the perception of facial emotional expressions. Twelve healthy male volunteers completed two experimental sessions each, in a randomized, balanced order, double-blind design. A single oral dose of escitalopram (10 mg) or placebo was administered $3 \mathrm{~h}$ before the task. Participants were presented to a task composed of six basic emotions (anger, disgust, fear, happiness, sadness, and surprise) that were morphed between neutral and each standard emotion in $10 \%$ steps. Escitalopram facilitated the recognition of sadness and inhibited the recognition of happiness in male, but not female faces. No drug effect on subjective measures was detected. These results confirm that serotonin modulates the recognition of emotional faces, and suggest that the gender of the face can have a role in this modulation. Further studies including female volunteers are needed.
\end{abstract}

Key words: Serotonin; Escitalopram; Facial expression; Emotion; Serotonin reuptake inhibitor

\section{Introduction}

Selective serotonin reuptake inhibitor (SSRI) agents have been widely and successfully used for the pharmacological treatment of several psychiatric disorders, including depressive and anxiety disorders, and the clinical efficacy of SSRIs has been attributed to an enhancement of 5-hydroxytryptamine (5-HT) neurotransmission (1). Nevertheless, little is known about the effect of such drugs on the neural mechanisms underlying cognitive and emotional processing.

Facial expressions of basic emotions have been used for the study of emotional processing, particularly the affective information processing, which is an initial step related to the search for signals of biological relevance in the environment. This processing is likely to be impaired in some psychiatric disorders and, in this regard, cognitive theories link negative distortions in information processing to the onset and/or maintenance of depression and anxiety (2). In addition, it has been proposed that antidepressant drugs work, at least in part, by reducing a negative bias in perception and memory that is believed to initiate and/or maintain symptoms of depression and anxiety (3).
Several studies have shown that changes of 5-HT neurotransmission can modulate the identification of emotional faces, particularly fearful faces (reviewed in Ref. 4). Briefly, there is evidence showing that the reduction of the availability of the precursor of 5-HT synthesis, tryptophan, impairs the recognition of facial expressions of fear by healthy female, but not male volunteers, without any effect on the recognition of other emotional faces. On the other hand, the acute dietary supplementation of tryptophan increases the identification of happiness and fear by female healthy volunteers. Citalopram has been the most used SSRI as a challenge for assessing 5-HT functions, probably due to its relatively mild side effects and availability for intravenous administration. Reported evidence has shown that single intravenous administration of $10 \mathrm{mg}$ citalopram facilitates the recognition of fearful and happy faces by healthy female volunteers, and a single oral administration of $20 \mathrm{mg}$ citalopram has a similar effect on the recognition of fearful faces by healthy volunteers of both genders. In contrast, daily oral administration of $20 \mathrm{mg}$ of the drug for 7 days has been shown to impair the identification of facial expressions

Correspondence: W.C. Alves-Neto, Rua Tenente Catão Roxo, 2750, 14051-140 Ribeirão Preto, SP, Brasil.

E-mail:wolmeneto@hotmail.com

Received August 25, 2009. Accepted February 3, 2010. Available online March 5, 2010. Published March 12, 2010. 
of fear, anger and disgust by healthy female volunteers.

In the present study, we used escitalopram, the pharmacologically active S-enantiomer of RS-citalopram, since the R-enantiomer has been shown to reduce the effects of the S-enantiomer (5), probably due to negative allosteric interaction at the level of the 5-HT transporter. As a result, escitalopram behaves as a highly potent and selective ligand of the 5-HT transporter and, accordingly, clinical studies have shown that escitalopram causes few side effects and has a relatively fast onset of action (6).

In view of the reduced number of studies with male participants, and data from our laboratory pointing to the influence of the phases of the menstrual cycle on emotional processing (7) we initially tested only healthy male volunteers. To control for the possible influence of individual variability on emotional processing, we used a crossover design, assuming that intra-subject comparisons would allow the detection of subtle drug effects, which could be masked by individual variability.

Therefore, the objective of this study was to evaluate the effect of a single 10-mg dose of the SSRI escitalopram on the identification of basic emotional faces by healthy male volunteers. In view of previous results showing an increase of the recognition of fearful faces induced by a single dose of other antidepressants, we hypothesized that escitalopram would facilitate the recognition of fear and other negative expressions.

\section{Material and Methods}

\section{Participants}

The sample was composed of 12 healthy male volunteers aged 21 to 27 years ( mean $=24.14$ years; $S D=2.17$ ), evaluated twice in a crossover design (24 experimental sessions).

Psychiatric diagnoses were excluded by the Portuguese version of the Structured Clinical Interview for DSM-IV, clinical version (SCID-CV) (8). General medical conditions were also excluded by clinical history, physical examination and laboratory tests. Misuse of psychoactive substances was excluded by medical history and urinary drug screening. The volunteers were free of any medication for at least 2 months before the procedures. Trait anxiety, measured by the State-TraitAnxiety Inventory Trait form (STAI-T) (9) was low (mean $=29.33$; SD $=4.14$ ), varying from 22 to 37 .

The study was approved by the Ethics Committee of the Faculdade de Medicina de Ribeirão Preto, Universidade de São Paulo, and written informed consent was obtained from all volunteers.

\section{Subjective measures}

Subjective states were evaluated by the Portuguese translation (10) of the Visual Analogue Mood Scale - VAMS (11). This scale is composed of 16 items divided into 4 factors denoted anxiety, sedation, cognitive impairment, and discomfort. For each item, the subject was requested to mark a point on a $100-\mathrm{mm}$ line between two adjectives of opposite meaning, which represented the present feeling. Physical symptoms that can be related to subjective anxiety were detected by the Bodily Symptoms Scale - BSS (10). The intensity of each symptom is graded from 0 (no symptom) to 4 (highest). The total BSS score was the sum of the values of all the items for each patient.

\section{Facial expression recognition task}

Faces from the Pictures of Facial Affect Series (12) portraying six basic emotions (anger, disgust, fear, happiness, sadness, and surprise) had been morphed between neutral $(0 \%)$ and each standard emotion (100\%), in 10\% steps of emotion intensity. For each emotion, 4 pictures, 2 males and 2 females, were presented for each intensity level, comprising 40 stimuli for each emotion. The faces were presented on the computer screen for $0.5 \mathrm{~s}$, with an interval (blank screen) of $4.5 \mathrm{~s}$ between each stimuli. Volunteers were asked to choose the response that best described the emotion in the picture and to register their responses as soon as possible by pressing one of the labeled keys on the keyboard.

\section{Procedures}

Participants were evaluated twice in sessions separated by at least 2 weeks, in a randomized, balanced order, double-blind, placebo-controlled, and crossover design. They attended the laboratory between 9:00 and 12:00 am after a light breakfast. Subjective measures (VAMS and BSS) were taken four times: 1) 20 min after arriving to the laboratory and just before the capsule intake; 2) $2 \mathrm{~h}$ after taking the capsule; 3 ) immediately before starting the face recognition task (described above), and 4) just after finishing the task.

Oral escitalopram $(10 \mathrm{mg})$ or placebo was given $3 \mathrm{~h}$ before the task. Placebo and escitalopram capsules were prepared by a pharmacist who was unaware of the experimental design. Five volunteers were given placebo in the first experimental session and escitalopram in the second; 7 volunteers were given escitalopram in the first and placebo in the second experimental session. Neither the participant nor the researcher was aware of the contents of the capsule taken during the experimental sessions.

\section{Data analysis}

Data were analyzed using the SPSS statistical package version 13.0. The area under the curve for the proportion of correct answers for each intensity level of the emotion (10 steps) for each emotion and for both treatments was calculated as an index of individual accuracy in the performance in the task and analyzed using repeated measures MANOVA (Hotteling's Trace). An initial analysis of possible effects of the repetition of the experiment was carried out considering as within-subject factors the experimental 
session order (first and second) and emotion type (anger, disgust, fear, sadness, surprise, and happiness). The drug treatment effect was analyzed considering the factors emotion (6), gender of the face (male and female) and treatment (escitalopram or placebo). Significant interactions were followed by paired $t$-tests. Subjective measures were also analyzed using repeated measures MANOVA, considering the factors time (4) and treatment (2).

\section{Results}

\section{Subjective measures}

No significant effect of drug treatment was found in the VAMS subjective measures (anxiety factor $F(1,10)=0.19$; $\mathrm{P}=0.669$; sedation $\mathrm{F}(1,10)=0.28 ; \mathrm{P}=0.606$; cognitive impairment $F(1,10)=1.16 ; P=0.307$; discomfort $F(1,10)$ $=0.01 ; P=0.913)$ or in the BSS global index $(F(1,10)=$ $0.15 ; P=0.707)$. Independent of treatment, the volunteers reported an increase of subjective anxiety $(F(1,10)=4.54$; $P=0.025)$ and of physical symptoms $(F(1,10)=2.43 ; P=$ $0.001)$ during the experimental session. in female faces $(t=2.75 ;$ d.f. $=11 ; P=0.019)$.

\section{Discussion}

The main finding of the present study is that escitalopram enhances the identification of sadness and reduces the identification of happiness, but the effect occurs mainly when the participant views male faces. Two issues arise: how does escitalopram enhance perception of sadness and reduce happiness perception, and why are males more sensitive to this effect when viewing male rather than female images?

The opposite effects of a single administration of escitalopram on the recognition of sad and happy faces shown by the present results resemble the pattern of response observed in depressive disorders. Briefly, depressive patients have been shown to preferentially attend to sad facial expressions over simultaneously presented neutral facial expressions and interpret emotionally neutral faces as sad faces. This bias seems to be specific to cues signaling sadness, since there is no evidence for preferential allocation

\section{Performance in the facial recognition task}

No effect of the repetition of the experimental session was observed (order of the experimental session factor $F(1,10)=0.33 ; P=0.578$ ).

A single dose of escitalopram (10 $\mathrm{mg}$ ) modulated the recognition of facial emotional expressions depending on the emotion evaluated (emotion and treatment interaction, $F(5,7)=9.65 ; \mathrm{P}=$ $0.005)$. Post hoc analyses (paired $t$-test) revealed that escitalopram increased the identification of sad faces $(t=5.50$; d.f. $=11 ; P<0.001)$, and tended to increase recognition of angry faces $(\mathrm{t}=$ 1.89 ; d.f. $=11 ; P=0.085)$, as well as to impair identification of surprise $(t=2.11$; d.f. $=11 ; P=0.058)$ and happiness $(t=$ $2.04 ;$ d.f. $=11 ; P=0.067$ ).

There was also an influence of the gender of the face on the effect of treatment (emotion, treatment and face) gender interaction $(F(5,7)=15.84 ; P$ $=0.001)$. As shown in Figure 1, escitalopram improved the identification of sadness in male faces $(t=8.97$; d.f. $=$ 11 ; $P<0.001)$, without any effect on female faces $(t=0.62 ;$ d.f. $=11 ; P=$ 0.546). The drug also had opposite effects on happy faces, impairing the recognition in male faces $(t=5.39$; d.f. $=11 ; \mathrm{P}<0.001)$, whereas improving it
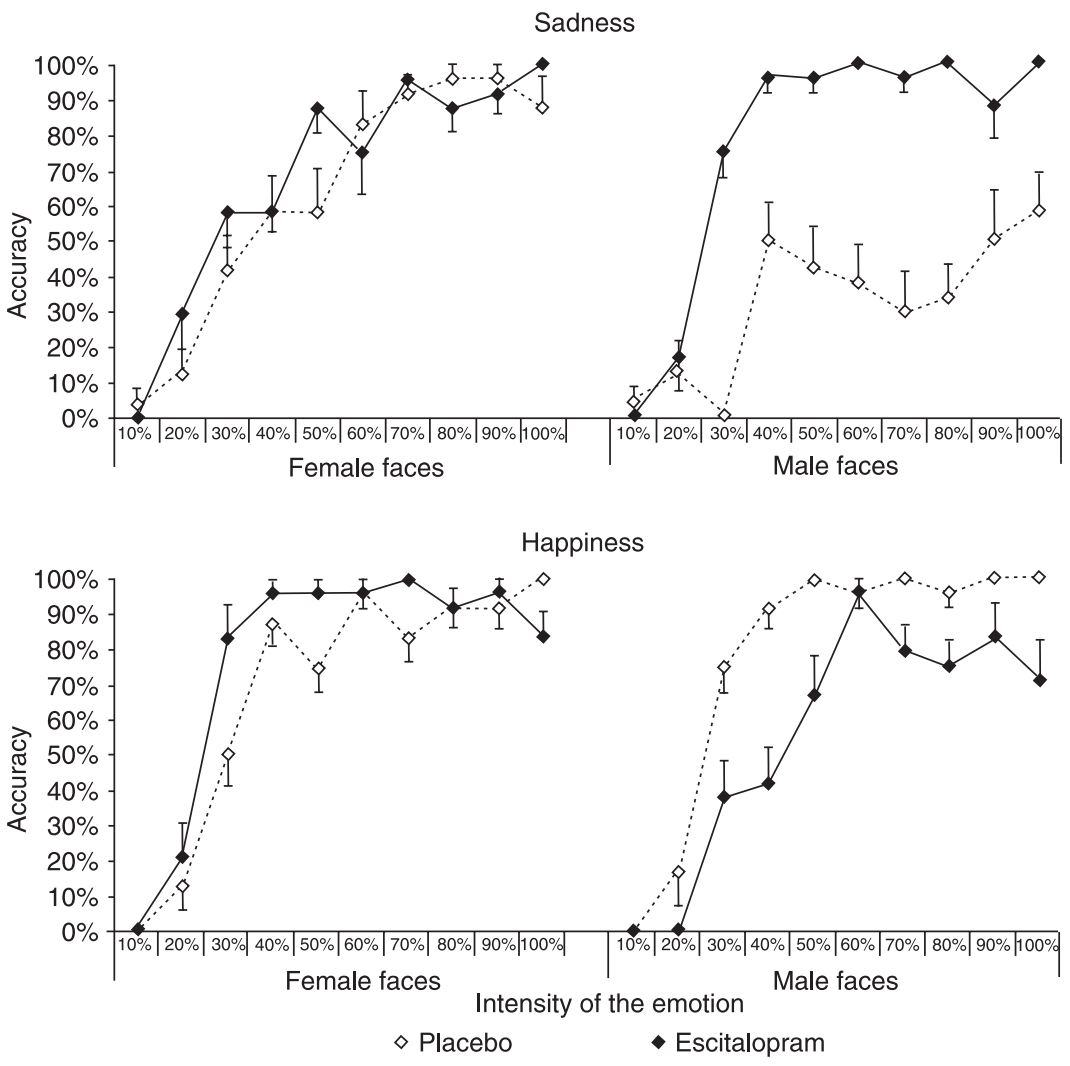

Figure 1. Accuracy of the identification of emotional faces. Effect of a single dose of escitalopram (10 $\mathrm{mg}$ ) on the accuracy in the identification of sadness (upper panel) and happiness (bottom panel) on female and male faces, as a function of face emotion intensity levels (in 10\% steps), by 12 male healthy volunteers evaluated twice, in a double-blind placebo-controlled crossover design. Data are reported as means \pm SD. 
of attention to cues that signal other negative emotions. Although less robust, there is also evidence pointing to the hypothesis that responsiveness to positive emotional cues would be blunted in depressive patients (13). Hence, the behavioral effect of acute escitalopram may be considered to be a "depressive effect", and this interpretation is consistent with the clinical evidence on delayed symptom improvement following antidepressant administration and the widely accepted view of an impaired 5-HT functioning in depression (3).

However, the interpretation of the effect of a single dose of SSRIs on 5-HT function is not straightforward. Some microdialysis studies in animals have shown increases in cortical extracellular 5-HT following acute SSRI administration (see, e.g., Ref. 14), but others have shown a greater increase of extracellular 5-HT in the raphe nuclei than in the neocortex (15). Therefore, it is possible that low acute doses of SSRIs preferentially increase $5-\mathrm{HT}$ concentration near the cell bodies of serotonergic neurons, reducing their firing rate due to the activation of somatodendritic $5-\mathrm{HT}_{1 \mathrm{~A}}$ autoreceptors, thus decreasing 5-HT release and lowering synaptic 5-HT concentration postsynaptically. According to these arguments, the opposite effects of a single administration of escitalopram on the recognition of sad and happy faces shown by the present results suggest a decrease of serotonergic transmission.

In this regard, we have recently shown that a single dose of escitalopram given to healthy male volunteers prolonged the fear provoked by the simulated public speaking test (16). This result resembles the early reported effect of a 5-HT2 antagonist in the same test, thus suggesting a decreased functioning of 5-HT neurotransmission. In contrast, escitalopram increased plasma cortisol and prolactin levels immediately after the speech, suggesting an increased $5-\mathrm{HT}$ function (16). This paradox may be solved by taking into account animal studies showing that the same pharmacological probe could differentially affect distinct subpopulations of 5-HT neurons (17), probably due to a balance between presynaptic (inhibitory) and postsynaptic (excitatory) actions, depending on the kind of the environmental stimulus. In this direction, previous data have shown that the manipulation of the 5-HT system through pharmacological challenges can have different effects depending on the emotion evaluated. Of significance for the present study is the opposite effect of tryptophan depletion on sadness and fearfulness observed in healthy participants, i.e., decreasing the identification of fearful faces, but increasing the recognition of sad faces (Deakin $B$, personal communication).

Previous reports, predominantly regarding female participants, found that acute administration of citalopram mainly increases the recognition of facial expressions of fear (4). However, in this study no effect of escitalopram on fear detection was observed. Pharmacodynamic or pharmacokinetic properties of escitalopram could be re- sponsible for these differences. Although escitalopram is the S-enantiomer of the racemic compound citalopram, many studies indicate that the former is more potent and has a more rapid onset of action than the latter (5), and SPECT studies have shown that escitalopram has a higher serotonin transporter occupancy compared with citalopram (18). Thus, these differences may also contribute to the discrepancy between the present results with escitalopram and the results reported with citalopram.

Another factor that could have contributed to our results is the process of selection of the participants. We were very rigorous in the exclusion of any psychiatric disorder through semi-structured interviews conducted by skilled clinical psychiatrists. These procedures led to a very homogenous sample, with low anxiety personality traits. Reported results indicate that individuals with high scores of trait-anxiety recognize threatening stimuli, including fearful faces, better than individuals with low trait-anxiety (19). High levels of neuroticism are associated with increased processing of negative information in emotional categorization tasks and a higher threshold for identifying happiness in facial expressions (20). Thus, personality characteristics can determine individual differences in emotional recognition tasks, which have been taken into account by the crossover experimental design adopted in the first experiment and the inclusion just of volunteers with low levels of anxiety.

We also found the impairment of the identification of happy faces, another result that is apparently divergent from reported findings showing a facilitating effect on the recognition of happiness of acute SSRIs (3). Actually, when we analyzed the gender of the faces we found that escitalopram impaired the recognition of male faces, but facilitated the identification of happiness in female faces. Therefore, the inconsistency between the present and previously reported results may be due to the gender of the recognized faces, a factor that has not been evaluated in former studies.

That escitalopram-modulated perception of sadness and happiness in faces of the same gender as the viewer may reflect a general sensitivity to emotion in faces of one's own gender. Yet, previous studies have not considered the interactions between gender of the viewer and gender of the faces, which restrains further comparisons.

Finally, the present lack of effect of escitalopram on subjective anxiety suggests that the serotonergic modulation of facial expression processing can occur independently of changes in the conscious recognition of the feelings aroused by emotional faces and independently of changes in mood states. This points to a specific effect on early preconscious cognitive processing that occurs early in the course of treatment and may be related to later clinical improvement. Since we did not use standard instruments to evaluate depressive state during the procedures, we cannot discard the possibility that the facilitation of sadness recognition and impairment of happiness recognition would 
be accompanied by changes in subjective depressive states. Further studies must address this issue.

The results obtained in this study need to be appreciated with caution, in view of the small size of the sample, possible bias created by the repetition of the experimental session and the fact that we studied only male volunteers. Further studies including female volunteers are needed to understand the interaction between gender of the viewer and gender of the face. In spite of these shortcomings, we have shown that a single dose of the SSRI escitalopram was able to affect the recognition of basic emotions in facial expressions by healthy male volunteers, supporting a 5-HT modulation of the recognition of emotional faces. In addi-

\section{References}

1. Deakin JF. The role of serotonin in panic, anxiety and depression. Int Clin Psychopharmacol 1998; 13 (Suppl 4): S1-S5.

2. Beck AT. Cognitive therapy of depression. The Guilford clinical psychology and psychotherapy series. New York: Guilford Press; 1979.

3. Harmer CJ, Bhagwagar Z, Perrett DI, Vollm BA, Cowen PJ, Goodwin GM. Acute SSRI administration affects the processing of social cues in healthy volunteers. Neuropsychopharmacology 2003; 28: 148-152.

4. Del-Ben CM, Ferreira CA, Alves-Neto WC, Graeff FG. Serotonergic modulation of face-emotion recognition. Braz J Med Biol Res 2008; 41: 263-269.

5. Sanchez C. The pharmacology of citalopram enantiomers: the antagonism by R-citalopram on the effect of S-citalopram. Basic Clin Pharmacol Toxicol 2006; 99: 91-95.

6. Waugh J, Goa KL. Escitalopram: a review of its use in the management of major depressive and anxiety disorders. CNS Drugs 2003; 17: 343-362.

7. Guapo VG, Graeff FG, Zani AC, Labate CM, dos Reis RM, Del-Ben CM. Effects of sex hormonal levels and phases of the menstrual cycle in the processing of emotional faces. Psychoneuroendocrinology 2009; 34: 1087-1094.

8. First MB. Structured Clinical Interview for DSM-IV axis I disorders: SCID-I: clinician version: administration booklet. Washington: American Psychiatric Press; 1997.

9. Spielberger CD. Manual for the State/Trait Anxiety Inventory (form Y): (self evaluation questionnaire). Palo Alto: Consulting Psychologists Press; 1983.

10. Zuardi AW, Guimaraes FS, Moreira AC. Effect of cannabidiol on plasma prolactin, growth hormone and cortisol in human volunteers. Braz J Med Biol Res 1993; 26: 213-217.

11. Norris $\mathrm{H}$. The action of sedatives on brain stem oculomotor tion, the present results justify the use of escitalopram as a pharmacological tool for exploring the role of $5-\mathrm{HT}$ in the normal emotional processing and in the pathophysiology of anxiety and depressive disorders.

\section{Acknowledgments}

Research supported by CNPq (\#554490/2005-6). F.G. Graeff is supported by a research fellowship from Fundação de Amparo ao Ensino, Pesquisa e Assistência do Hospital das Clínicas de Ribeirão Preto (FAEPA\#04/05) and CNPq. C.M. Del-Ben and V.G. Guapo are supported by research fellowships from CNPq. systems in man. Neuropharmacology 1971; 10: 181-191.

12. Ekman P, Friesen WV. Pictures of facial affect. Palo Alto: Consulting Psychology; 1976.

13. Leppanen JM. Emotional information processing in mood disorders: a review of behavioral and neuroimaging findings. Curr Opin Psychiatry 2006; 19: 34-39.

14. David DJ, Bourin M, Jego G, Przybylski C, Jolliet P, Gardier AM. Effects of acute treatment with paroxetine, citalopram and venlafaxine in vivo on noradrenaline and serotonin outflow: a microdialysis study in Swiss mice. Br J Pharmacol 2003; 140: 1128-1136.

15. Bel N, Artigas F. Fluvoxamine preferentially increases extracellular 5-hydroxytryptamine in the raphe nuclei: an in vivo microdialysis study. Eur J Pharmacol 1992; 229: 101-103.

16. Garcia-Leal C, Del-Ben C, Leal F, Graeff F, Guimaraes F. Escitalopram prolonged fear induced by simulated public speaking and released hypothalamic-pituitary-adrenal axis activation. J Psychopharmacol 2009 (in press).

17. Lowry CA, Johnson PL, Hay-Schmidt A, Mikkelsen J, Shekhar A. Modulation of anxiety circuits by serotonergic systems. Stress 2005; 8: 233-246.

18. Klein N, Sacher J, Geiss-Granadia T, Mossaheb N, Attarbaschi $\mathrm{T}$, Lanzenberger R, et al. Higher serotonin transporter occupancy after multiple dose administration of escitalopram compared to citalopram: an [123I]ADAM SPECT study. Psychopharmacology 2007; 191: 333-339.

19. Surcinelli P, Codispoti M, Montebarocci O, Rossi N, Baldaro B. Facial emotion recognition in trait anxiety. J Anxiety Disord 2006; 20: 110-117.

20. Chan SW, Goodwin GM, Harmer CJ. Highly neurotic neverdepressed students have negative biases in information processing. Psychol Med 2007; 37: 1281-1291. 